\title{
1 Intricate structure of the interphase chromocenter revealed by the 2 analysis of a factor involved in species formation
}

3 Natalia Y. Kochanova ${ }^{1}$, Tamas Schauer ${ }^{2}$, Grusha Primal Mathias ${ }^{3}$, Andrea Lukacs ${ }^{1}$, Andreas

4 Schmidt ${ }^{4}$, Andrew Flatley ${ }^{5}$, Aloys Schepers ${ }^{5}$, Andreas W. Thomae ${ }^{3}$ and Axel Imhof ${ }^{1,4^{*}}$

$5 \quad{ }^{1}$ Biomedical Center, Chromatin Proteomics Group, Department of Molecular Biology, Faculty of

6 Medicine, Ludwig-Maximilians-Universität München, Großhaderner Strasse 9, 82152 Planegg-

7 Martinsried, Germany.

8 'Biomedical Center, Bioinformatics Core Facility, Ludwig-Maximilians-Universität München,

9 Großhaderner Strasse 9, 82152 Planegg-Martinsried, Germany.

$10{ }^{3}$ Biomedical Center, Core Facility Bioimaging, Ludwig-Maximilians-Universität München,

11 Großhaderner Strasse 9, 82152 Planegg-Martinsried, Germany

12 'Biomedical Center, Protein Analysis Unit, Faculty of Medicine, Ludwig-Maximilians-Universität

13 München, Großhaderner Strasse 9, 82152 Planegg-Martinsried, Germany.

$14{ }^{5}$ Helmholtz Zentrum München, Deutsches Forschungszentrum für Gesundheit und Umwelt (GmbH), 15 Institute for Diabetes and Obesity, Monoclonal Antibody Core Facility and Research Group

16 Ingolstädter Landstr. 1, 85764 Neuherberg, Germany

17

18 * To whom correspondence should be addressed. Tel: +49 89 218075420; Fax: +49 89 218075410;

19 Email: imhof@Imu.de 
ABSTRACT

In higher eukaryotes centromeres often coalesce into a large intranuclear domain called the chromocenter. Chromocenters are important for the organization of pericentric heterochromatin and a disturbance of their formation results in an upregulation of repetitive elements and causes defects in chromosome segregation. Mutations in the gene encoding for the centromere associated Drosophila speciation factor HMR show very similar phenotypes suggesting a role of HMR in chromocenter architecture and function. We performed confocal and super resolution microscopy as well as proximity based biotinylation experiments of HMR and its associated protein HP1a to generate a molecular map of HMR and HP1a bound chromatin. Our work reveals an intricate internal structure of the centromeric chromatin region, which suggests a role of HMR in separating heterochromatin from centromeric chromatin.

\section{INTRODUCTION}

In many eukaryotes pericentromeric and centromeric chromatin of multiple chromosomes cluster in interphase to form a nuclear domain, the so-called chromocenter (1-3). The chromocenters are composed of pericentromeric heterochromatin and CenpA containing centromeric chromatin, which are both rich in repetitive DNA and evolutionarily highly dynamic $(4,5)$. They form and are held together by multiple components ranging from RNA transcribed from the repeats (6) over DNA binding factors (7), protein-protein interactions (8) to histone posttranslational modifications (9). Interference with chromocenter formation results in an upregulation of transposable elements, mitotic defects and the formation of micronuclei $(7,8)$. Despite their functional importance, the centromere as well as the pericentromeric repeats are highly divergent with regard to size, sequence and protein composition even in very closely related species $(4,10,11)$. This rapid divergence of centromeric sequences is thought to be accompanied by an adaptive evolution of centromere binding proteins to counteract a meiotic drive, which would otherwise result in the potentially deleterious expansion of centromeric repeats $(5,12)$. This hypothesis is supported by the fact that many of the gene products that result in hybrid incompatibility are either proteins that bind to the centromeric or pericentromeric heterochromatin (13-16) or RNA molecules that localize in this region $(17,18)$. One of the best characterised hybrid incompatibility factors is the Hybrid male rescue protein HMR (19). HMR interacts with the heterochromatin protein HP1a and co-localizes with the centromere-specific $\mathrm{H} 3$ variant dCenpA (CID) in Drosophila cell lines and imaginal disc cells (13). HMR binding is also observed at several euchromatic sites where it colocalizes with known boundary factors (20). Interestingly, the intracellular localization of HMR varies among different tissues. In interphase cells of larval brains it is primarily found at pericentromeric heterochromatin $(15,21,22)$ whereas it also associates with telomere structures on salivary gland polytene chromosomes $(13,22)$. Mutations of $H m r$ results in an upregulation of transposable elements $(13,23)$ and an increase in mitotic defects (13). As this phenotype is very similar to defects upon knockdown of nucleoplasmin (NIp), which interacts with HMR and plays a role in centromere clustering $(8,13)$, we wondered about HMR's role in chromocenter architecture. To investigate the intricate structure of the Drosophila chromocenters we performed confocal and super resolution microscopy using antibodies directed against HP1a, 
1 HMR and dCenpA and determined the proteome in proximity of HMR and HP1a using APEX2 based

2 proximity biotinylation. Our results suggest that HMR is located at boundaries between HP1a

3 containing heterochromatin and centromeric or transcriptionally active chromatin. Besides the

4 proximity to heterochromatic and known centromeric factors we also observe a close proximity of

5 HMR to nucleolar proteins, transcription factors, nuclear pore components and the condensin and

6 cohesin complex. These findings suggest an important role of HMR in orchestrating the formation of

7 the evolutionarily very dynamic chromocenter domain. As a consequence, the differential regulation of

8 HMR in different species of Drosophila results in complex mitotic defects in hybrid animals containing

9 two different and separately evolved genomes.

\section{RESULTS}

\section{HMR and dCenpA form an interdigitated centromeric network}

To confirm the previously detected centromeric localization of HMR in Drosophila cells with another antibody, we performed immunofluorescent staining using a FLAG antibody in a cell line where HMR is endogenously tagged with the FLAG epitope at the C-terminus using CRISPR/Cas9 (20). Consistent with our previous results (13) most of the FLAG signals co-localize with the signal we obtained when using an anti-dCenpA antibody, which confirms the close proximity of HMR and centromeric chromatin during interphase (Figure 1A). Notably, the quantitation of more than 120 interphase centromeres indicated that $57 \%$ of all centromere foci overlap with HMR foci. This suggests that the localization of HMR to the centromere is either cell cycle regulated or specific for a subset of centromeres (Figure 1B), which may explain the differences observed when staining HMR in different tissues $(13,15)$. Another observation that is difficult to reconcile with the clustered staining of HMR in cells is the fact that ChIP-seq data suggest HMR binding along the euchromatic arms often at boundaries between HP1a containing heterochromatin and actively transcribed genes (20). As centromeres are also transcribed (10) and in proximity to pericentromeric heterochromatin, we wondered if HMR might also localize to a putative boundary between these two different chromatin regions. Due to its repetitive nature, pericentromeric as well as centromeric chromatin is difficult to map in ChIP-seq experiments. We therefore investigated the interphase centromere and its surrounding chromatin using super resolution STED microscopy. These experiments revealed that the chromocenter is not homogenously formed by dCenpA containing chromatin but constitutes a structural meshwork of interdigitated dCenpA and HMR proteins (Fig. 1C, Movie S1), which we assume to constitute an intranuclear domain frequently defined as the chromocenter (24). In agreement to previous results (25), this centromeric region is surrounded by large lobes of HP1a containing heterochromatin, which are often bordered by HMR signals (Supplemental Fig. S1, Movie S1). This intricate structure is further confirmed by super resolution microscopy stainings of dCenpA and $\mathrm{dCenpC}$ as well as HMR and $\mathrm{dCenpC}$. These co-stainings revealed a substantial colocalization of $\mathrm{dCenpC}$ with $\mathrm{dCenpA}$, in contrast to the shifted localization observed between HMR and dCenpC or dCenpA (Fig. 1D). 
To further unravel the details of this architectural meshwork at the chromocenter, we generated stable cell lines expressing HMR and HP1a fused to an engineered ascorbate peroxidase from soybean (APEX2) under a copper inducible promoter to perform proximity biotinylation (Fig. 2A). Upon treatment of APEX2 expressing cells with biotin phenol and hydrogen peroxide a localized burst of diffusible biotin-phenoxyl radicals is generated. These radicals then react with nearby $(<20 \mathrm{~nm})$ electron rich amino acid side chains leading to the biotinylation of neighboring proteins that can be subsequently purified and identified using shot gun mass spectrometry (26-32). We confirmed the expression of ectopic proteins by Western blotting using antibodies against HMR, HP1a and APEX2 (Fig. 2B). The APEX2 protein was fused to a double nuclear localization signal to determine the nontargeted nuclear proteome. To perform the biotinylation reaction at physiological protein levels, we used non-inducing conditions for $\mathrm{HMR}_{\mathrm{AP}}$ expression but induced the expression of HP1 $\mathrm{a}_{\mathrm{AP}}$ (Fig. 2B and C). For the APEX2NLS cell line we added copper sulfate to the medium before the biotinylation to induce the expression of APEX2NLS and to match HP1 $\mathrm{a}_{\mathrm{AP}}$ expression levels. At the expression level used, $\mathrm{HMR}_{\mathrm{AP}}$ localizes to centromeres, marked by the centromere-specific histone variant dCenpA, HP1 $1 a_{A P}$ occupies a domain in the nucleus, which coincides with endogenous HP1a staining and APEX $X_{N L S}$ localizes to the nucleus (Fig.2B), showing a proper nuclear localization of the fusion proteins under the conditions used.

\section{HMR and HP1a APEX2 fusion proteins biotinylate defined nuclear domains}

To analyze the localization of the biotinylation by the expressed fusion proteins, we stained the cell lines for APEX2 and biotin after performing an in situ biotinylation reaction (Fig. 3A). Consistent with the limited diffusion of the phenoxy radicals generated by APEX2, we observed a strong biotinylation signal colocalizing with the APEX2-fusion proteins (Fig. 3B). Cells that do not express nuclear APEX2 or cells that were not treated with biotin-phenol and hydrogen peroxide only showed background biotinylation signals. As the different cell lines expressed different levels of the APEX2-fusion protein and as HP1a has previously been shown to be very dynamic, we adjusted the concentration of biotinphenol and the duration of hydrogen peroxide treatment to maintain proper localization while achieving a high degree of biotinylation of the neighboring proteome. While HMRAP cells treated for 25 minutes with hydrogen peroxide at a high concentration of biotin-phenol $(5 \mathrm{mM})$ showed a highly localized biotinylation (Supplemental Fig. S2), a similar treatment of HP1aAP cells resulted in a broader biotinylation signal even though a reduced biotin-phenol concentration was used $(0.5 \mathrm{mM})$ (Supplemental Figs. S2 and S3). This broader signal was not observed when HP1 $\mathrm{a}_{\mathrm{AP}}$ cells were treated for only 5 minutes with 0.5 mM biotin-phenol (Supplemental Fig. S3).

\section{Proteomic analysis of HMR and HP1a containing domains}

Upon in vivo biotinylation, we compared the individual proteomes from cell lines expressing $H_{M} R_{A P}$, HP1 $1_{A P}$ and APEX $X_{N L S}$ that were either treated with biotin-phenol and $\mathrm{H}_{2} \mathrm{O}_{2}$ or with DMSO only (Figure 4A). Using label free quantitation and a statistical analysis of the proximity-based proteome from at least 4 biological replicates we identified 406,300 and 349 proteins that were specifically biotinylated in HMR AP, HP1 $_{A P P}$ and APEX $X_{N L S}$ expressing cells respectively (Supplementary Tab. S2). A 
comparative analysis of the isolated proximity proteomes revealed a strong overlap between the

HP1 $1 a_{A P}$ and the APEX $X_{N L S}$ proteome $(71 \%)$, which was not the case for HMR AP $_{\text {a }}$ and APEX $(44 \%)$. The latter finding may reflect the lower expression level of HMR, the lower mobility and the more specific localization of HMR $\mathrm{AP}_{\mathrm{AP}}$ compared to HP1aAP (Fig. 3B). To further characterize the different proteomic composition within the three proximity proteomes we compared the enriched GO terms using the Gene Ontology Consortium tool (http://www.geneontology.org) (Supplementary Tab. S3). Consistent with its known function we found the GO terms such as chromatin silencing, histone modification and positive regulation of chromatin organization for HP1 $\mathrm{a}_{A P}$. For HMRAP, we found the GO terms heterochromatin organization involved in chromatin silencing, telomere maintenance, mitotic sister chromatid segregation and nucleus organization, which were consistent with the observed phenotypes of HMR mutations in Drosophila melanogaster (13, 15, 22, 33, 34). This proximity-based proteome also strengthens the hypothesis that HMR localizes in between dCenpA and HP1a containing chromatin as components of both domains are found in proximity of HMR. Proteins that are biotinylated by all three factors were often highly abundant nuclear factors constituting the splicing machinery or structural proteins such as NLP, D1 or Lamin, which are likely to be in close proximity to most nuclear factors. As the long hydrogen peroxide treatment resulted in a lower HP1 $\mathrm{a}_{\mathrm{AP}}$ staining upon biotinylation, we also measured the HP1 $\mathrm{a}_{\mathrm{AP}}$ proximity proteome upon 5 minutes of biotinylation. Due to the shorter biotinylation time we identified less specifically biotinylated proteins (122). When comparing the proteins biotinylated after 5 minutes peroxide treatment with the ones isolated after 25 minutes of biotinylation we identified a higher percentage of previously characterized HP1a interactors after 25 minutes of biotinylation $(35,36)$ (Supplementary Fig. S4) suggesting that a longer biotinylation reaction covers a broader range of HP1aAP containing domains. To identify proteins that specifically localize close to HMRAP and HP1 $\mathrm{aAP}_{A P}$ and are not distributed throughout the entire nucleus, we selected proteins that were preferentially biotinylated in the HMRAP or the HP1 1aAP cell line but not or only to a much lesser degree in the APEXNLS line (Supplementary Tab. S2). As the HP1 aAP specific proximity proteome showed a large overlap with the proteome in proximity to APEX $X_{N L S}$, only very few chromatin associated factors were significantly closer to HP1aAP than to APEXNLS (Supplementary Table S1), which prevented us from further analyzing the HP1aAP proximity proteome in great depth. For HMRAP, however we detected a substantial number of proteins being much closer to HMRAP than to APEX $X_{N L S}$ and therefore displayed the proximity-based proteome of HMRAP in the form of a network graph. In this graph individual proteins are displayed as nodes and previously published interactions as edges (Fig. 4C). Besides a large fraction of nucleolar proteins, we find the condensin and subunits of the cohesin complex, a large fraction of nuclear pore proteins, several insulator proteins, as well as a network of transcription factors including the previously identified hybrid incompatibility factor GFZF, as well as nucleosome remodeling factors and histonemodifying enzymes (Fig. 4C).

\section{Cohesin and condensin factors reside in proximity to HMR}

38 We were particularly intrigued by the proximity of $\mathrm{HMR}_{\mathrm{AP}}$ to cohesin and condesin subunits as it has 
chromosome condensation and integrity reminiscent of those observed in cells depleted of condensins or showing abnormal accumulation of cohesins (15). To validate the proximity of HMR with members of the cohesin complex, we compared the ChIP-sequencing profiles of HMR in S2 cells (20), the cohesin subunit Rad21 (vtd) and the condensin subunit CAP-H2 in Kc167 cells (37). Consistent with the proximity labelling result, approximately half (51\%) of the HMR binding sites colocalize with binding sites of the cohesin complex and $20 \%$ with the CAP-H2 subunit of the condensin complex (Fig. 5A). The analysis of the genomic features of the HMR binding sites that overlap with Rad21 or CAP-H2 demonstrated that the HMR binding sites colocalizing with condensin or cohesin are more frequently found at promoter regions (Fig. 5B, left panel). By classifying chromatin domains using the five-state chromatin model (38) the HMR binding sites that colocalize with the condensin subunit CAP-H2 were more frequently found at active chromatin or HP1a containing heterochromatin (Fig. 5B, right panel).

To simulate the hybrid situation where increased levels of the complex containing HMR and LHR are found (13), we compared a HMR ChIP-seq analysis upon overexpression of HMR and LHR (39) with the condensin and cohesin profiles. Upon overexpression we detected 1314 additional HMR peaks, many of them colocalizing with binding sites of CAP-H2 (Fig. 5C). When we aligned ChIP-seq profiles along HMR binding sites (both native and induced), we found, that CAP-H2 ChIP enrichment is greater at HMR sites having high HP1a signals or at HMR sites appearing only upon overexpression (Fig. 5D). Rad21 ChIP enrichment in contrast is non-discriminating for classes of HMR binding. Architectural proteins, such as condensins and cohesins, were shown to cluster at TAD boundaries (37). We therefore wondered, whether HMR binding sites also reside there. Interestingly, HMR binding sites have local minima in TAD separation score (40) (Figs. 5D and E) suggesting that HMR localizes and potentially functions at genomic sites important for the 3D organization of chromatin.

\section{DISCUSSION}

Interspecies hybrids are either sterile or lethal due to the incompatibility of their genomes $(41,42)$ or the incompatibility of the oocyte cytoplasm with a conflicting parental genome. Crosses between the closely related species Drosophila melanogaster and Drosophila simulans result in hybrid male lethality dependent on the presence of $D$. mel HMR and its interaction partner LHR from $D$. sim. More recently, gfzf, a gene encoding an essential FLWYTCH domain containing protein residing on the $3^{\text {rd }}$ chromosome of $D$.sim, has also been shown to contribute to male hybrid lethality (43). Though the effect of these factors on hybrid male lethality is well described, their molecular function is still largely unknown. HMR has been implicated in several cellular processes such as the silencing of transposable elements $(13,22,48)$ or the faithful chromosome segregation and sister chromatid separation $(13,15,33)$. HMR forms distinct foci that frequently overlap with the centromere in $D$. mel cell lines as well as in imaginal discs derived from D.mel larvae. An $\mathrm{Hmr}$ transgene carrying a mutation that affects its centromeric localization fails to kill male hybrids, suggesting that HMR's mode of chromatin binding is causally related to the male lethality phenotype $(13,34)$. Our systematic microscopic analysis reveals that while most of the HMR foci colocalize with dCenpA this is not always the case. This suggests either a cell cycle regulated HMR-centromere interaction or a 
chromosome specific binding of HMR similar to the SATIII RNA, which has also been implicated in species formation (44) and only associates with chromosomes 2,3 and X (18). The overall localization of HMR may also be dependent on the cell type as we for example do not observe sizeable telomeric localization of HMR in S2 cells, while it is substantial in polytene chromosomes (13). Super resolution microscopy of the HMR containing nuclear domains furthermore revealed that HMR, dCenpA and dCenpC partially overlap within a nuclear domain that is clearly separated from HP1a containing heterochromatin. We interpret these findings as an indication of HMRs role in separating individual chromatin domains.

Within many organisms centromeres, pericentromeric heterochromatin and in some cases even telomeres cluster to form a nuclear domain or aggregate called chromocenter (24). These aggregates are frequently found at the nuclear periphery or around the nucleolus. Strikingly, we find HMR in proximity of nucleolar components and members of the nuclear pore suggesting that it might function in positioning the chromocenter within the nucleus after cell division.

Besides these structural components of the nucleus, the HMR proximity proteome also reveals its close localization to factors that separate functional chromatin domains. This is consistent with previous ChIP-seq mapping of HMR binding sites, which showed that a subset of HMR peaks border HP1a containing chromatin at promoters of active genes (20). Many of these HMR binding sites also colocalize with a condensin complex, two subunits of which we also find in close proximity to HMR. The proximity of HMR with condensin and cohesin at sites that separate different chromatin domains is consistent with the findings of Blum and colleagues (15) who described the Hmr phenotype as reminiscent to perturbations in cohesin or condensin function with frequent anaphase breakages at boundaries between eu- and heterochromatin. The striking discrepancy between the number of HMR binding sites observed in ChIP-seq experiments and the few histologically visible clusters suggest that HMR binding sites cluster in vivo at the chromocenter (Fig. 6). Such a role of clustered boundary regions in organizing nuclear 3D structure has also been observed in human as well as Drosophila cells $(45,46)$ suggesting a common mechanism for TAD organization. These domains not only play important function in the regulation of gene expression but also in reassembling the nuclear structure during the mitotic telophase. Increased levels of HMR, which is also observed in hybrids, results in an accumulation of HMR binding at previously unbound CAP-H2 binding sites which may in turn result in the formation of multiple novel clusters and a dysfunctional organization of the nucleus (47). In summary, our results suggest that HMR is, in conjunction with a highly complex molecular machinery, involved in organizing the nuclear 3D structure. A failure of this tight regulation could then interfere with this well-orchestrated and self-organized assembly process leading to a catastrophic outcome such as hybrid lethality. 
2 Cloning. APEX fusions were cloned into PMT vector (13), which was cut with Xbal and Notl. GST-

3 APEX was cloned into pGEX-6P-1 vector (13), cut with EcoRI and Notl. Cloning was performed with In-Fusion cloning kit (Clontech). The list of primers is available in the supplementary table S1.

5 Cell culture, transfection and generation of stable cell lines. Drosophila L2-4 cells (13) were grown in Schneider medium supplemented with $10 \%$ fetal calf serum, penicillin and streptomycin at $26^{\circ} \mathrm{C}$. To generate a stable cell line, 3-4 million of cells were transfected with $2 \mu \mathrm{g}$ of plasmid mixed with XtremeGENE HP (Roche) transfection reagent according to manufacturer's instructions. After transfection, cells were selected for 3 weeks with Hygromycin B (Invitrogen) at $100 \mu \mathrm{g} / \mathrm{ml}$. Optional induction of cell lines with $250 \mu \mathrm{M} \mathrm{CuSO} 4$ was performed 12-24 hours before experiments.

APEX proximity biotinylation, nuclear extraction and immunoprecipitation. For biotinylation cells were grown in roller bottles (Greiner) to density of $5^{*} 10^{6} \mathrm{cells} / \mathrm{ml}$. For biotinylation in solution $5^{*} 10^{8}$ $10^{9}$ cells were resuspended in $200 \mathrm{ml}$ biotin-phenol/PBS or DMSO/PBS and incubated for $30 \mathrm{~min}$ at room temperature (RT). $\mathrm{H}_{2} \mathrm{O}_{2}$ was added to biotin-phenol treated cells to the concentration of $1 \mathrm{mM}$ and cells were pelleted for $20 \mathrm{~min}$ at $250 \mathrm{~g}$. The supernatant was aspirated, and cells were washed three times in quenching solution (10 mM sodium azide, $10 \mathrm{mM}$ sodium ascorbate and $5 \mathrm{mM}$ Trolox in PBS) or (untreated cells) in PBS. The last washing step was performed in $15 \mathrm{ml}$ falcons. Nuclei isolation and extraction was performed as in (48) with modifications. All buffers were supplemented with freshly added protease inhibitors. Cells were swelled in 3 packed cell volumes (PCV) (e.g. 2,1 $\mathrm{ml}$ ) of hypotonic buffer (10 mM Tris pH 7.6, $10 \mathrm{mM} \mathrm{NaCl}, 1.5 \mathrm{mM} \mathrm{MgCl}$, $0.1 \mathrm{mM}$ EDTA) on ice for 30 min. Cells were centrifuged $10 \mathrm{~min} 250 \mathrm{~g}$, and the pellet was resuspended in $3 \mathrm{PCV}$ of hypotonic buffer supplemented with $0,2 \% \mathrm{NP}-40$. Cells were rotated for $10 \mathrm{~min}$ at $4^{\circ} \mathrm{C}$ for the lysis of plasma membrane. Nuclei were pelleted at $1000 \mathrm{~g}$ for $10 \mathrm{~min}$ and washed with $2 \mathrm{ml}$ of quenching solution supplemented with protease inhibitors. After the wash nuclei were centrifuged for 10 min with $1500 \mathrm{~g}$ and the nuclear pellet was snap-frozen in liquid nitrogen. Nuclei were resuspended in $3 \mathrm{ml}$ Tris-Ex100 buffer (10 mM Tris pH 7.6, $100 \mathrm{mM} \mathrm{NaCl}, 1.5 \mathrm{mM} \mathrm{MgCl}, 0.5 \mathrm{mM}$ EGTA and 10\% v/v glycerol) + 1500 units Mnase +1500 units Benzonase $+2 \mathrm{mM} \mathrm{CaCl}_{2}$. Chromatin was digested for $20 \mathrm{~min}$ at $26^{\circ} \mathrm{C}$ and the reaction was stopped by addition of EDTA and EGTA to $10 \mathrm{mM}$ on ice. Nuclei were disrupted by Dounce homogenization using 10 strokes with a tight-fitting pestle. For chromatin extraction and solubilization, $\mathrm{NaCl}$ (to $600 \mathrm{mM}$ ) and detergents (Triton X-100 to $1 \%$, sodiumdeoxycholate (SOD) to $0.5 \%$ and SDS to $0.1 \%$ ) were added and chromatin was incubated for $1 \mathrm{~h}$ at $4^{\circ} \mathrm{C}$. Nuclear extracts were cleared by centrifugation $20 \mathrm{~min} 10000 \mathrm{~g}$ and dialyzed for 4 hours at $4^{\circ} \mathrm{C}$ through $3.5 \mathrm{MWCO}$ Millipore membranes against Tris-Ex100 with detergents and without glycerol, supplemented with 0.2 $\mathrm{mM}$ PMSF and $1 \mathrm{mM}$ DTT. The obtained nuclear extract was snap-frozen. For streptavidin purification, nuclear extract with freshly added protease inhibitors was mixed with $500 \mu$ of Pierce streptavidin magnetic beads, prewashed two times in Tris-Ex100 buffer with detergents. Immunoprecipitation was performed for 1,5 hours at RT. Beads were washed two times with TrisEx100 buffer with detergents (with protease inhibitors), once with $2 \mathrm{M}$ Urea $10 \mathrm{mM}$ Tris, and again twice with Tris-Ex100 with detergents and protease inhibitors. 
1 For biotinylation on plates, $800 \mathrm{ml}$ of $5^{*} 10^{9}$ million/ml cells were adhered on $4015-\mathrm{cm}$ plates for mammalian cells for 1 hour. Cells were incubated in biotin-phenol/PBS (20 plates) or DMSO/PBS (20 plates) for $30 \mathrm{~min}$, and $\mathrm{H}_{2} \mathrm{O}_{2}$ was added to biotin-phenol treated cells to the concentration of $1 \mathrm{mM}$ for $5 \mathrm{~min}$. Solution was aspirated and quenching solution (or PBS for untreated cells) was added. Cells were scraped off, washed one more time in quenching solution and subjected to nuclear extraction as described above.

Mass spectrometry. Beads from APEX pulldowns were washed 3 times with $500 \mu \mathrm{l}$ of $50 \mathrm{mM}$ Tris $\mathrm{pH} \mathrm{7.5,} \mathrm{4M}$ urea, and on-bead digestion was performed. Reduction was completed in $500 \mu \mathrm{l}$ of $20 \mathrm{mM}$ DTT in $50 \mathrm{mM}$ Tris, $2 \mathrm{M}$ urea $\mathrm{pH} 7.5$ with Lys $\mathrm{C} 450 \mathrm{ng} /$ sample at $27^{\circ} \mathrm{C}$ for $1 \mathrm{~h}$. Alkylation followed with $50 \mathrm{mM}$ iodoacetamide for $1 \mathrm{~h} 25^{\circ} \mathrm{C}$, shaking $900 \mathrm{rpm}$, and was stopped by addition of DTT to $10 \mathrm{mM}$ final concentration. The samples were incubated at $25^{\circ} \mathrm{C}$ shaking $900 \mathrm{rpm}$ for 2 more hours. $300 \mu \mathrm{l}$ water was added to reduce urea concentration to $1.5 \mathrm{M}$, and $1.5 \mu \mathrm{g}$ trypsin and $2 \mathrm{mM}$ final concentration $\mathrm{CaCl}_{2}$ were added for overnight incubation shaking $900 \mathrm{rpm}$. In the morning another 1.5 $\mu \mathrm{g}$ of trypsin was added, and the sample was incubated for another 4 hours while shaking. After collection of the supernatant, the beads were washed 2 times with $100 \mu \mathrm{l}$ of $20 \mathrm{mM}$ Tris $50 \mathrm{mM} \mathrm{NaCl}$ $25 \% \mathrm{ACN}$ in order to elute loosely-bound tryptic peptides from the beads. Washes were combined with supernatant, evaporated at less than $28^{\circ} \mathrm{C}$, and desalted. The second elution of remaining peptides from the beads was performed with $300 \mu \mathrm{l} 0.05 \%$ SDS, $0.1 \%$ formic acid (FA) at $80^{\circ} \mathrm{C}$ for 10 min. The second elution was evaporated and subjected to HILIC chromatography. After desalting and HILIC both elutions were combined, evaporated and resuspended in $45 \mu \mathrm{l} 0.1 \%$ FA. Peptide mixtures were subjected to nanoRP-LC-MS/MS analysis on an Ultimate 3000 nano chromatography system coupled to a QExactive HF mass spectrometer (both Thermo Fisher Scientific) in 2-4 technical replicates $(5 \mu \mathrm{l}$ each). The samples were directly injected in $0.1 \%$ FA onto the separating column (120 x $0.075 \mathrm{~mm}$, in house packed with ReprosilAQ-C18, Dr. Maisch $\mathrm{GmbH}, 2.4 \mu \mathrm{m}$ ) at a flow rate of 0.3 $\mu \mathrm{l} / \mathrm{min}$. The peptides were separated by a linear gradient from $3 \% \mathrm{ACN}$ to $40 \% \mathrm{ACN}$ in $50 \mathrm{~min}$. The outlet of the column served as electrospray ionization emitter to transfer the peptide ions directly into the mass spectrometer. The QExactive was operated in a TOP10 duty cycle, detecting intact peptide ion in positive ion mode in the initial survey scan at 60,000 resolution and selecting up to 10 precursors per cycle for individual fragmentation analysis. Therefore, precursor ions with charge state between $2+$ and $5+$ were isolated in a 2 Da window and subjected to higher-energy collisional fragmentation in the HCD-Trap. After MS/MS acquisition precursors were excluded from MS/MS analysis for 20 seconds to reduce data redundancy. Siloxane signals were used for internal calibration of mass spectra.

Protein MaxQuant search. The raw data files of APEX pulldowns were analysed with MaxQuant version 1.5.3.12 against dmel-all-translation-r6.08.fasta database from Flybase. All the parameters were set to default except choosing "Match between runs“ and LFQ and iBAQ quantitations. Technical replicates were assigned to one experiment (biological replicate).

Data sources. ChIP-seq datasets were available from GEO with the following accession numbers: GSE86106 (HMR native and HP1a), GSE118291 (HMR induced), GSE54529 (Rad21 and CAPH2). 
5-state chromatin domains were taken from (43) from which "yellow" and "red" were merged to active domains. Genomic regions (promoters, exons etc.) are based on the Flybase annotation (version r6.17). TAD separation scores derived from http://chorogenome.iefreiburg.mpg.de/data_sources.html. Genomic coordinates from the dm3 release were converted to dm6 using the liftover tool from UCSC.

ChIP-seq analysis. Raw reads were aligned to the reference genome (UCSC dm6) using bowtie2 (version 2.2.9) and quality filtered by samtools (version 1.3.1). ChIP-seq profiles were created by Homer (version 4.9) and normalized by the number of reads and the corresponding input. Peaks were called by Homer with parameters -style factor -F 2 -size 200 (except for Rad21 -F 4). Peak overlaps were plotted as Venn diagrams using the Vennerable R package (version 3.1.0.9). HMR peaks were sub-grouped by the overlap with CAPH2 or Rad21 peaks. Such peak groups were characterized by genomic features (promoters, exons, etc.) and chromatin domains (active, etc.) by taking the size of the intersecting genomic ranges between the peaks and each feature and dividing by the sum of the intersecting range sizes. ChIP-seq profiles were aligned to the pool of native and induced HMR peaks and visualized as heatmaps or as average composite plots. Heatmaps were ordered by HMR native enrichment and grouped by HP1a enrichment. Figures were created by R base graphics.

Mass spectrometry analysis. Log2-transformed LFQ values were imputed with the impute.MinProb function (imputeLCMD R package v2.0) with parameter $q=0.05$ and normalized by median normalization. Moderated t-statistics were computed by fitting a linear model with empirical Bayes moderation using the limma R package (version 3.34.9). The data were visualized as volcano plots using $\mathrm{R}$ graphics. Code is available upon request. GO-term analysis was done using Gene Ontology Consortium (http://geneontology.org) tool excluding redundant GO terms. The protein-protein interaction network was visualized using Cytoscape. Protein-protein interaction data were taken from the STRING database (49) using experiments, databases, gene fusion and co-occurrence as interaction sources and minimum required interaction score of 0.9 . Additional interactions were taken from the database Flybase and indicated as dotted lines.

Immunofluorescent staining. Cells were adhered on coverslips for $30 \mathrm{~min}$, washed with PBS and fixed in $0.3 \%$ Triton X-100/3.7\% formaldehyde/PBS for 12 min at RT. After wash with PBS cells were permeabilized in $0.25 \%$ Triton/PBS on ice for $6 \mathrm{~min}$. Cells were rinsed 2 times and washed again twice with PBS, following blocking in Image-iT FX signal enhancer (Invitrogen) for 45 min at RT. Primary antibodies, diluted in 5\% normal goat serum (NGS) (Dianova), were incubated with coverslips $1 \mathrm{~h}$ at $\mathrm{RT}$ (or at $4^{\circ} \mathrm{C}$ overnight for high resolution microscopy). Coverslips were washed with $0.1 \%$ Triton $\mathrm{X}$ 100/PBS and PBS and incubated with secondary antibody in 5\% NGS $1 \mathrm{~h}$ at RT. Following a wash in $0.1 \%$ Triton X-100/PBS and 2 washes in PBS, cells were stained in DAPI/PBS (200 ng/mL or 50 $\mathrm{ng} / \mathrm{mL}$ for high resolution microscopy) and washed again in PBS. Cells were mounted in VECTASHIELD (Vector Labs) or ProLong ${ }^{\text {TM }}$ Diamond Antifade (Thermo Fisher Scientific), for epifluorescence and confocal or super resolution microscopy, respectively. 
1 For biotinylation followed by immunofluorescence on coverslips, $10^{6}$ cells was adhered on coverslips

2 for $30 \mathrm{~min}$, incubated with biotin-phenol/PBS or DMSO/PBS for $30 \mathrm{~min}$, followed by (optional) addition

3 of $1 \mathrm{mM} \mathrm{H}_{2} \mathrm{O}_{2}$ for denoted time. Cells were next washed in quenching solution and subjected to

4 immunofluorescent staining as described above. Biotinylation in solution was performed with $10^{6}$ cells in $200 \mu \mathrm{l}$ biotin-phenol/PBS or DMSO/PBS and $1 \mathrm{mM} \mathrm{H}_{2} \mathrm{O}_{2}$, added for denoted time. Cells were pelleted during biotinylation $250 \mathrm{~g}$, resuspended in $200 \mu \mathrm{l}$ quenching solution, adhered on coverslips for $15 \mathrm{~min}$ and processed for IF as described above.

Image acquisition was performed on a Zeiss Axiovert 200 epifluorescence microscope with a CCD Camera (AxioCam MR, Zeiss). Confocal microscopy was performed th the core facility bioimaging of the Biomedical Center with an inverted Leica SP8X WLL microscope, equipped with $405 \mathrm{~nm}$ laser, WLL2 laser (470 - $670 \mathrm{~nm}$ ) and acousto-optical beam splitter. Gated-STED Images were acquired with a 100x1.4 objective, image pixel size was $24-25 \mathrm{~nm}$. The following fluorescence settings were used: DAPI (excitation 405 nm; emission 415-470 nm), Alexa Fluor 594 (590 nm; 600-625) and Abberior STAR 635P (635; 645-720). Recording was done line sequentially to avoid bleed-through and channel misalignment because of drift. Signals were recorded with hybrid photo detectors (HyDs) in counting mode. STED and confocal images were deconvolved using Huygens 17.10 p2. All images were processed using ImageJ.

Western blotting of whole cell extracts. 15 million cells from each cell line were collected, washed two times in PBS and resuspended on ice in $80 \mu$ RIPA buffer with freshly added protease inhibitors and 30 units benzonase. Lysates were left on ice for $30 \mathrm{~min}$, and afterwards $20 \mu \mathrm{l}$ of $5 \mathrm{x}$ Laemmli buffer was added. Lysates were boiled $10 \mathrm{~min} 96^{\circ} \mathrm{C}$ before loading on the gel $(10 \mu \mathrm{l})$.

Antibodies. For immunofluorescence and western blotting, rat anti-HMR 2C10 antibody (Helmholtz Zentrum München, (13)) was used in dilution 1:25 (or 1:5 for high resolution microscopy); rat antidCenpA 7A2 (Helmholtz Zentrum München, (8)) 1:100 (or 1:50 for high resolution microscopy); rabbit anti-dCenpA (Immunofluorescence grade, Actif Motif) 1:500 (or 1:250 for high resolution microscopy); mouse anti-HP1a C1A9 (50) 1:100; anti-APEX2 20H10 (raised in this study, Helmholtz Zentrum München) 1:50; Streptavidin-Alexa555 (Thermo Fisher Scientific) 1:400; rabbit anti-histone H3 (Abcam) 1:3000, mouse anti-FLAG (Sigma M2, 1mg/ml) 1:100. Rabbit anti-dCenpC antibody was kindly provided by Christian Lehner and used in dilution 1:5000 (1:1000 for high resolution microscopy).

Antibody generation. Wistar rats were immunized subcutaneously (s.c.) and intraperitonially (i.p.)

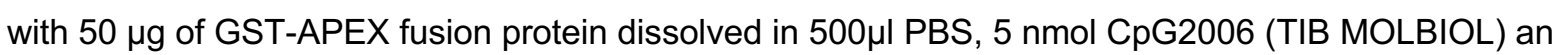
equal volume of incomplete Freund's adjuvant. 6 weeks after immunization a $50 \mu \mathrm{g}$ boost injection was applied i.p. and s.c. three days before fusion. Fusion of the splenic B cells and the myeloma cell line P3X63Ag8.653 was performed using polyethylene glycol 1500 according to standard protocols (51). Hybridoma supernatants were tested by solid-phase enzyme-linked immunoassay (ELISA) using the recombinant GST-fusion protein and verified by Western blotting of whole cell extracts from APEX2 fusions-expressing cell lines (Fig. 2B). Hybridoma cell line from specifically reacting 
supernatants were cloned twice by limiting dilution. Experiments in this study were performed with clone $20 \mathrm{H} 10$ (rat $\lg \mathrm{G} 2 \mathrm{a} / \mathrm{k}$ ).

\section{DATA AVAILABILITY}

The mass spectrometry proteomics data have been deposited to the ProteomeXchange Consortium via the PRIDE (52) partner repository with the dataset identifier PXD011310.

\section{ACKNOWLEDGEMENT}

We thank the Christian Lehner and Sarah Elgin for the Rabbit anti dCenpC and the mouse anti-HP1a antibody respectively. We furthermore would like to thank Nitin Phadnis and Patrick Heun for critical comments on the manuscript as well as as Irene Vetter for cloning the HP1a-APEX2 fusion construct, Ignasi Forne for mass-spectrometry analysis, Tobias Straub for initial bioinformatic analysis and the entire Imhof group/Becker department for helpful discussions.

\section{FUNDING}

This work was supported by the grants from the Deutsche Forschungsgemeinschaft (DFG) to Al (CIPSM and AI23/9-1), NK and AL (Graduate School of Quantitative Biosciences Munich (QBM)). Funding for open access charges: DFG CRC 1064/Z03

\section{CONFLICT OF INTEREST}

The authors state no conflict of interest

\section{REFERENCES}

1. Jones,K.W. (1970) Chromosomal and nuclear location of mouse satellite DNA in individual cells. Nature, 225, 912-915.

2. Fransz,P., De Jong,J.H., Lysak,M., Castiglione,M.R. and Schubert,I. (2002) Interphase chromosomes in Arabidopsis are organized as well defined chromocenters from which euchromatin loops emanate. Proc Natl Acad Sci USA, 99, 14584-14589.

3. Jones,K.W. and Robertson,F.W. (1970) Localisation of reiterated nucleotide sequences in Drosophila and mouse by in situ hybridisation of complementary RNA. Chromosoma, 31, 331345.

4. Jagannathan,M., Warsinger-Pepe,N., Watase,G.J. and Yamashita,Y.M. (2017) Comparative Analysis of Satellite DNA in the Drosophila melanogasterSpecies Complex. G3 (Bethesda), 7 , 693-704.

5. Malik,H.S. and Henikoff,S. (2009) Major evolutionary transitions in centromere complexity. Cell, 138, 1067-1082.

6. Probst,A.V., Okamoto,I., Casanova,M., Marjou,El,F., Le Baccon,P. and Almouzni,G. (2010) A Strand-Specific Burst in Transcription of Pericentric Satellites Is Required for Chromocenter Formation and Early Mouse Development. Dev Cell, 19, 625-638.

7. Jagannathan,M., Cummings,R. and Yamashita,Y.M. (2018) A conserved function for pericentromeric satellite DNA. eLife, 7. 
8. Padeken,J., Mendiburo,M.J., Chlamydas,S., Schwarz,H.-J., Kremmer,E. and Heun,P. (2013) The Nucleoplasmin Homolog NLP Mediates Centromere Clustering and Anchoring to the Nucleolus. Mol. Cell, 10.1016/j.molcel.2013.03.002.

9. Peters,A.H., O'Carroll,D., Scherthan,H., Mechtler,K., Sauer,S., Schöfer,C., Weipoltshammer,K., Pagani,M., Lachner,M., Kohlmaier,A., et al. (2001) Loss of the Suv39h histone methyltransferases impairs mammalian heterochromatin and genome stability. Cell, 107, 323337.

10. Talbert,P.B., Kasinathan,S. and Henikoff,S. (2018) Simple and Complex Centromeric Satellites in Drosophila Sibling Species. Genetics, 208, 977-990.

11. Clark,A.G., Eisen,M.B., Smith,D.R., Bergman,C.M., Oliver,B., Markow,T.A., Kaufman,T.C., Kellis,M., Gelbart,W., lyer,V.N., et al. (2007) Evolution of genes and genomes on the Drosophila phylogeny. Nature, 450, 203-218.

12. Henikoff,S. and Malik,H.S. (2002) Centromeres: selfish drivers. Nature, 417, 227-227.

13. Thomae,A.W., Schade,G.O.M., Padeken,J., Borath,M., Vetter,I., Kremmer,E., Heun,P. and Imhof,A. (2013) A Pair of Centromeric Proteins Mediates Reproductive Isolation in Drosophila Species. Dev Cell, 27, 412-424.

14. Ross,B.D., Rosin,L., Thomae,A.W., Hiatt,M.A., Vermaak,D., la Cruz,de,A.F.A., Imhof,A., Mellone,B.G. and Malik,H.S. (2013) Stepwise Evolution of Essential Centromere Function in a Drosophila Neogene. Science, 340, 1211-1214.

15. Blum,J.A., Bonaccorsi,S., Marzullo,M., Palumbo,V., Yamashita,Y.M., Barbash,D.A. and Gatti,M. (2017) The Hybrid Incompatibility Genes Lhr and Hmr Are Required for Sister Chromatid Detachment During Anaphase but Not for Centromere Function. Genetics, 10.1534/genetics.117.300390.

16. Bayes,J.J. and Malik,H.S. (2009) Altered Heterochromatin Binding by a Hybrid Sterility Protein in Drosophila Sibling Species. Science, 326, 1538-1541.

17. Ferree,P.M. and Prasad,S. (2012) How Can Satellite DNA Divergence Cause Reproductive Isolation? Let Us Count the Chromosomal Ways. Genetics Research International, 2012, 1-11.

18. Rošić,S., Köhler,F. and Erhardt,S. (2014) Repetitive centromeric satellite RNA is essential for kinetochore formation and cell division. J Cell Biol, 207, 335-349.

19. Barbash,D.A., Siino,D.F., Tarone,A.M. and Roote,J. (2003) A rapidly evolving MYB-related protein causes species isolation in Drosophila. Proc Natl Acad Sci USA, 100, 5302-5307.

20. Gerland,T.A., Sun,B., Smialowski,P., Lukacs,A., Thomae,A.W. and Imhof,A. (2017) The Drosophila speciation factor HMR localizes to genomic insulator sites. PLOS ONE, 12, e0171798.

21. Maheshwari,S. and Barbash,D.A. (2012) Cis-by-Trans Regulatory Divergence Causes the Asymmetric Lethal Effects of an Ancestral Hybrid Incompatibility Gene. PLoS Genet, 8, e1002597.

22. Satyaki,P.R.V., Cuykendall,T.N., Wei,K.H.-C., Brideau,N.J., Kwak,H., Aruna,S., Ferree,P.M., Ji,S. and Barbash,D.A. (2014) The hmr and Ihr hybrid incompatibility genes suppress a broad range of heterochromatic repeats. PLoS Genet, 10, e1004240.

23. Brideau,N.J. and Barbash,D.A. (2011) Functional conservation of the Drosophila hybrid incompatibility gene Lhr. BMC Evol Biol, 11, 57. 
24. Politz,J.C.R., Scalzo,D. and Groudine,M. (2013) Something Silent This Way Forms: The Functional Organization of the Repressive Nuclear Compartment. Annu. Rev. Cell. Dev. Biol., 29, 241-270.

25. Olszak,A.M., van Essen,D., Pereira,A.J., Diehl,S., Manke,T., Maiato,H., Saccani,S. and Heun,P. (2011) Heterochromatin boundaries are hotspots for de novo kinetochore formation. Nat Cell Biol, 13, 799-808.

26. Lam,S.S., Martell,J.D., Kamer,K.J., Deerinck,T.J., Ellisman,M.H., Mootha,V.K. and Ting,A.Y. (2015) Directed evolution of APEX2 for electron microscopy and proximity labeling. Nat Meth, 12, $51-54$.

27. Gao,X.D., Tu,L.-C., Mir,A., Rodriguez,T., Ding,Y., Leszyk,J., Dekker,J., Shaffer,S.A., Zhu,L.J., Wolfe,S.A., et al. (2018) C-BERST: defining subnuclear proteomic landscapes at genomic elements with dCas9-APEX2. Nat Meth, 15, 433-436.

28. Hung,V., Zou,P., Rhee,H.-W., Udeshi,N.D., Cracan,V., Svinkina,T., Carr,S.A., Mootha,V.K. and Ting,A.Y. (2014) Proteomic mapping of the human mitochondrial intermembrane space in live cells via ratiometric APEX tagging. Mol. Cell, 55, 332-341.

29. Rhee,H.-W., Zou,P., Udeshi,N.D., Martell,J.D., Mootha,V.K., Carr,S.A. and Ting,A.Y. (2013) Proteomic mapping of mitochondria in living cells via spatially restricted enzymatic tagging. Science, 339, 1328-1331.

30. Zuzow,N., Ghosh,A., Leonard,M., Liao,J., Yang,B. and Bennett,E.J. (2018) Mapping the mammalian ribosome quality control complex interactome using proximity labeling approaches. Mol. Biol. Cell, 29, 1258-1269.

31. Mick,D.U., Rodrigues,R.B., Leib,R.D., Adams,C.M., Chien,A.S., Gygi,S.P. and Nachury,M.V. (2015) Proteomics of Primary Cilia by Proximity Labeling. Dev Cell, 35, 497-512.

32. Gupta,R., Somyajit,K., Narita,T., Maskey,E., Stanlie,A., Kremer,M., Typas,D., Lammers,M., Mailand,N., Nussenzweig,A., et al. (2018) DNA Repair Network Analysis Reveals Shieldin as a Key Regulator of NHEJ and PARP Inhibitor Sensitivity. Cell, 10.1016/j.cell.2018.03.050.

33. Bolkan,B.J., Booker,R., Goldberg,M.L. and Barbash,D.A. (2007) Developmental and cell cycle progression defects in Drosophila hybrid males. Genetics, 177, 2233-2241.

34. Aruna,S., Flores,H.A. and Barbash,D.A. (2009) Reduced fertility of Drosophila melanogaster hybrid male rescue $(\mathrm{Hmr})$ mutant females is partially complemented by Hmr orthologs from sibling species. Genetics, 181, 1437-1450.

35. Alekseyenko,A.A., Gorchakov,A.A., Zee,B.M., Fuchs,S.M., Kharchenko,P.V. and Kuroda,M.I. (2014) Heterochromatin-associated interactions of Drosophila HP1a with dADD1, HIPP1, and repetitive RNAs. Genes Dev., 28, 1445-1460.

36. Swenson,J.M., Colmenares,S.U., Strom,A.R., Costes,S.V. and Karpen,G.H. (2016) The composition and organization of Drosophila heterochromatin are heterogeneous and dynamic. eLife, 5, 1445.

37. Van Bortle,K., Nichols,M.H., Li,L., Ong,C.-T., Takenaka,N., Qin,Z.S. and Corces,V.G. (2014) Insulator function and topological domain border strength scale with architectural protein occupancy. Genome Biol, 15, R82-18.

38. Filion,G.J., van Bemmel,J.G., Braunschweig,U., Talhout,W., Kind,J., Ward,L.D., Brugman,W., de Castro,I.J., Kerkhoven,R.M., Bussemaker,H.J., et al. (2010) Systematic protein location mapping reveals five principal chromatin types in Drosophila cells. Cell, 143, 212-224.

39. Cooper,J.C., Lukacs,A., Reich,S., Schauer,T., Imhof,A. and Phadnis,N. (2018) Altered chromatin localization of hybrid lethality proteins in Drosophila. bioRxiv, 10.1101/438432. 
40. Ramírez,F., Bhardwaj,V., Arrigoni,L., Lam,K.C., Grüning,B.A., Villaveces,J., Habermann,B., Akhtar,A. and Manke,T. (2018) High-resolution TADs reveal DNA sequences underlying genome organization in flies. Nat Commun, 9, 189.

41. Seehausen,O., Butlin,R.K., Keller,I., Wagner,C.E., Boughman,J.W., Hohenlohe,P.A., Peichel,C.L., Saetre,G.-P., Bank,C., Brännström,Å., et al. (2014) Genomics and the origin of species. Nat Rev Genet, 15, 176-192.

42. Presgraves,D.C. (2010) The molecular evolutionary basis of species formation. Nat Rev Genet, $11,175-180$.

43. Phadnis,N., Baker,E.P., Cooper,J.C., Frizzell,K.A., Hsieh,E., la Cruz,de,A.F.A., Shendure,J., Kitzman,J.O. and Malik,H.S. (2015) An essential cell cycle regulation gene causes hybrid inviability in Drosophila. Science, 350, 1552-1555.

44. Ferree,P.M. and Barbash,D.A. (2009) Species-Specific Heterochromatin Prevents Mitotic Chromosome Segregation to Cause Hybrid Lethality in Drosophila. PLoS Biol, 7, e1000234.

45. Gerasimova,T.I., Byrd,K. and Corces,V.G. (2000) A chromatin insulator determines the nuclear localization of DNA. Mol. Cell, 6, 1025-1035.

46. Yusufzai,T.M., Tagami,H., Nakatani,Y. and Felsenfeld,G. (2004) CTCF tethers an insulator to subnuclear sites, suggesting shared insulator mechanisms across species. Mol. Cell, 13, 291298.

47. van de Werken,H.J.G., Haan,J.C., Feodorova,Y., Bijos,D., Weuts,A., Theunis,K., Holwerda,S.J.B., Meuleman,W., Pagie,L., Thanisch,K., et al. (2017) Small chromosomal regions position themselves autonomously according to their chromatin class. Genome Res., 27, 922-933.

48. Barth,T.K., Schade,G.O.M., Schmidt,A., Vetter,I., Wirth,M., Heun,P., Imhof,A. and Thomae,A.W. (2015) Identification of Drosophila centromere associated proteins by quantitative affinity purification-mass spectrometry. Data Brief, 4, 544-550.

49. Szklarczyk,D., Morris,J.H., Cook,H., Kuhn,M., Wyder,S., Simonovic,M., Santos,A., Doncheva,N.T., Roth,A., Bork,P., et al. (2017) The STRING database in 2017: quality-controlled protein-protein association networks, made broadly accessible. Nucleic Acids Res, 45, D362D368.

50. James,T.C. and Elgin,S.C. (1986) Identification of a nonhistone chromosomal protein associated with heterochromatin in Drosophila melanogaster and its gene. Mol Cell Biol, 6, 3862-3872.

51. Köhler,G. and Milstein,C. (1975) Continuous cultures of fused cells secreting antibody of predefined specificity. Nature, 256, 495-497.

52. Vizcaíno,J.A., Deutsch,E.W., Wang,R., Csordas,A., Reisinger,F., Ríos,D., Dianes,J.A., Sun,Z., Farrah,T., Bandeira,N., et al. (2014) ProteomeXchange provides globally coordinated proteomics data submission and dissemination. Nat Biotechnol, 32, 223-226.

53. Heun,P., Erhardt,S., Blower,M.D., Weiss,S., Skora,A.D. and Karpen,G.H. (2006) Mislocalization of the Drosophila centromere-specific histone CID promotes formation of functional ectopic kinetochores. Dev Cell, 10, 303-315.

54. Van Bortle,K., Ramos,E., Takenaka,N., Yang,J., Wahi,J.E. and Corces,V.G. (2012) Drosophila CTCF tandemly aligns with other insulator proteins at the borders of $\mathrm{H} 3 \mathrm{~K} 27 \mathrm{me} 3$ domains. Genome Res., 22, 2176-2187. 
Figure 1: (A) - Staining of S2 FLAG-HMR line (20) with mouse anti-FLAG (Sigma), anti-HMR 2C10 and rabbit anti-dCenpA antibodies. (B) Quantification of centromeres overlapping with HMR. Experiments were performed in a dCenpA-GFP expressing cell line (53). In total 25 cells and 122 centromeres identified using an anti GFP antibody were counted. (C) Confocal (top panel) and stimulated emission depletion (STED) microscopy (middle panel) images of dCenpA and HMR. The bottom panel shows a zoom in of the centromeric region (D) Plot profiles of the highest fluorescence intensities of STED images of the chromocenter from S2 cells using anti dCenpA, anti dCenpC and anti HMR antibodies. Intensity graphs were built in ImageJ and normalized to one of the maximum peaks.

Figure 2: (A) APEX fusion constructs used for proximity based labeling of the chromocenter. (B) Western blots of whole cell lysates from stable cell lines, expressing HMRAP, HP1aA or APEX $\mathrm{A}_{\mathrm{NLS}}$ Antibodies used for Western blots were anti-HMR 2C10, anti-HP1a C1A9, anti-APEX2 $20 \mathrm{H} 10$ and rabbit anti-H3 (for loading control). (C) Localization of APEX fusions in respective stable cell lines. Antibodies used are the same as for Western blotting in (B). For cells with centromere staining (indicated by an asterisk) the inlet shows an approx. 2.3-fold zoom of the nucleus. Shown are the stainings of cells with (induced) or without (uninduced) copper mediated induction of the expression of the corresponding fusion protein.

Figure 3: (A) Schematic display of a proximity based biotinylation experiment. (B) Biotinylation experiment performed for 1 minute with $5 \mathrm{mM}$ biotin-phenol using the HMRAP cell line (top four panels) in the absence of biotin phenol and hydrogen peroxide, the presence of either biotin phenol or hydrogen peroxide or both reagents. The bottom panel shows a treatment of non-APEX expressing L2-4 cells treated with biotin phenol and hydrogen peroxide. For selected cells (indicated by an asterisk) the inlet shows an approx. 2.3-fold zoom of the nucleus. (C) Proximity based biotinylation of the HP1 $1 \mathrm{aAP}_{\mathrm{AP}}$ and the APEX $\mathrm{XLS}_{\mathrm{N}}$ cell lines using $0.5 \mathrm{mM}$ biotin-phenol. Stainings were performed with anti-APEX 20H10 antibody and anti-Streptavidin Alexa555. Scale bars represent $5 \mu \mathrm{m}$.

Figure 4: (A) Volcano plots of streptavidin pulldowns from extracts prepared from cells expressing

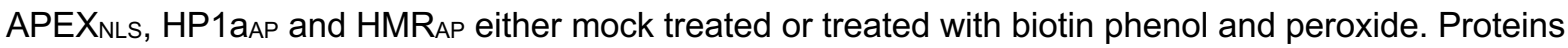
specifically biotinylated in the treated samples are indicated in red. (B) Venn diagram of proteins found enriched in the individual purifications. (C) Network diagram of proteins specifically enriched in HMR $_{\text {AP }}$ but not in APEX $X_{N L S}$. Solid lines were provided by the STRING databases using the highest stringency settings (0.9). Dotted lines were manually added based on information from Flybase.

Figure 5 (A) Peak overlaps of HMR, Rad21 and CAP-H2. (B) Distribution of HMR, HMR/Rad21 and HMR/CAP-H2 peaks across different genomic regions and chromatin types. (C) Peak overlaps of overexpressed HMR, Rad21 and CAP-H2. (D) Heatmaps of HMR (20), overexpressed HMR, HP1a (20), Rad21, CAP-H2 ChIP-seqs (54) and TAD separation score (40). All the peaks are centered at

(E) Composite plot of overexpressed HMR, 
1 Rad21, CAP-H2 ChIP-seq signals and TAD separation score centered to the HMR peaks' position.

2 Peaks that are only detected when HMR is overexpressed are indicated as new.

3 Figure 6: Model of organization of Drosophila interphase centromere. HMR and dCenpA protein 4 domains are interdigitating, and HMR domains are often bordering dCenpA domains from HP1a 5 domains. Some TAD boundaries, occupied by HMR, might be clustered at the border between centromeric and pericentric chromatins.

\section{SUPPLEMENTARY DATA}

8 Figure S1: Confocal microscopy images of L2-4 cells stained with rabbit anti-dCenpA, anti-HMR

$92 \mathrm{C} 10$ and anti-HP1a C1A9 antibodies. The highest intensities graph was built in ImageJ and normalized to one of the maximum peaks.

Figure S2: Biotinylation experiment performed for 25 minutes using the HMRAP, HP1aAP and APEXNLS cell lines. Controls are shown in the right panel. Stainings were performed with anti-APEX 20H10 antibody and anti-Streptavidin Alexa555. For selected cells (indicated by an asterisk) the inlet shows an approx. 2.3x zoom of the nucleus. Scale bars represent $5 \mu \mathrm{m}$. Exposure for APEX and biotin is indicated in white.

Figure S3: Time-course biotinylation experiment performed for the HP1aAP cell line. Stainings were performed with anti-APEX $20 \mathrm{H} 10$ antibody and anti-Streptavidin Alexa555. Scale bars represent 5 $\mu \mathrm{m}$.

Figure S4: Volcano plots of streptavidin pulldowns from extracts of cells expressing HP1aAP either mock treated or treated with biotin phenol and peroxide (for 5 and 25 minutes). Previously reported HP1a interactors, enriched in the pulldowns, are highlighted in green.

Table S1: List of primers used for cloning of Myc-HMR-APEX, Flag-HA-HP1a-APEX, Flag-HA-APEXNLS and GST-APEX constructs.

Table S2: Lists of proteins, enriched in HMR $\mathrm{AP}_{\mathrm{AP}}, \mathrm{HP1} \mathrm{a}_{A P}$ and APEX $X_{N L S}$ pulldowns from treated against

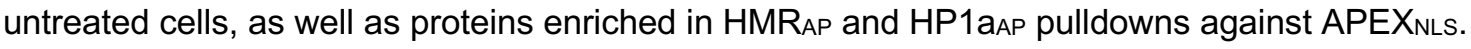

Table S3: GO term analysis of proteins enriched in $\mathrm{HMR}_{\mathrm{AP}}, \mathrm{HP} 1 \mathrm{a}_{\mathrm{AP}}$ and $\mathrm{APE} \mathrm{X}_{\mathrm{NLS}}$ pulldowns from

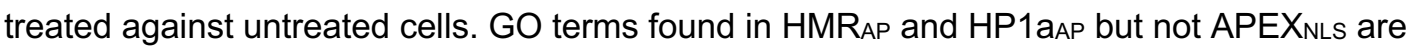
highlighted in light green.

Movie S1: 3D reconstruction from confocal HP1a (green) and super resolution STED HMR (red) and dCenpA (blue) microscopy. 
B
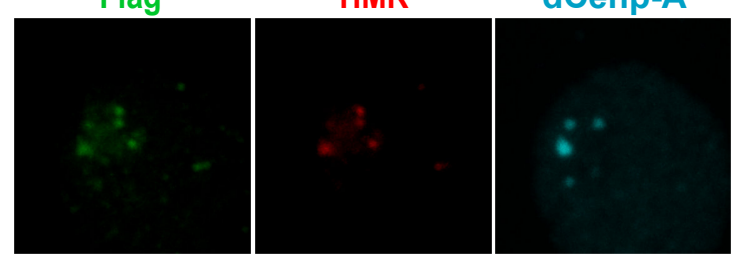

merge

merge

merge
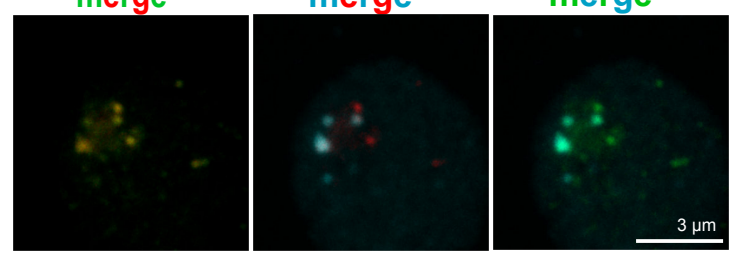

C

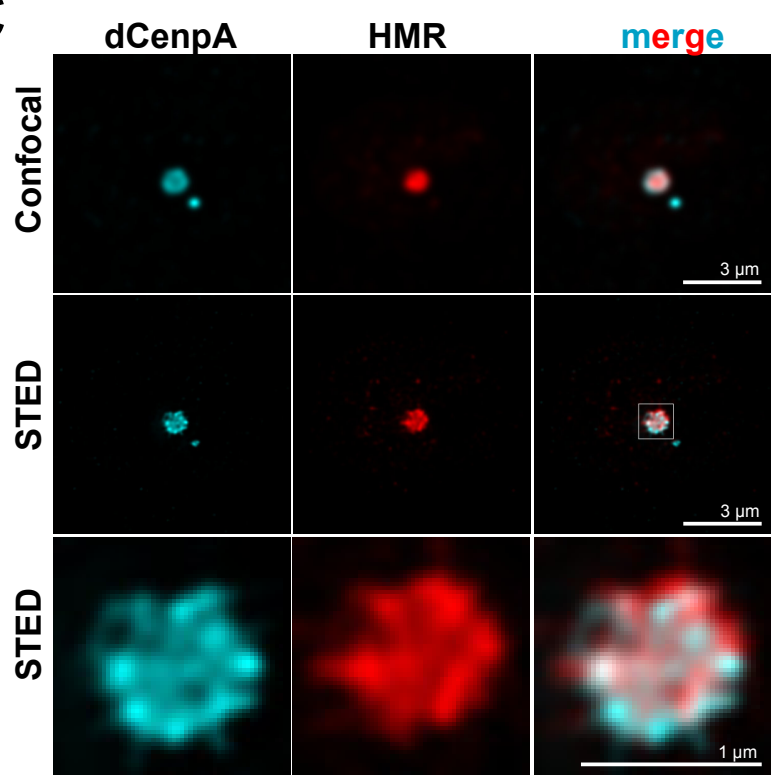

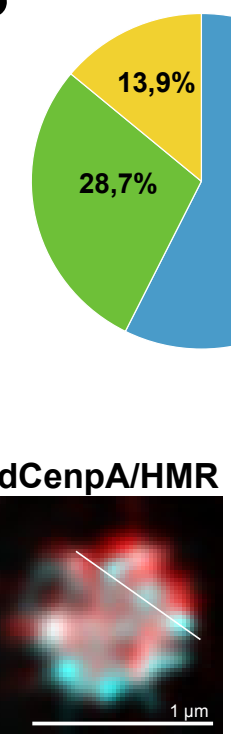

dCenpC/HMR
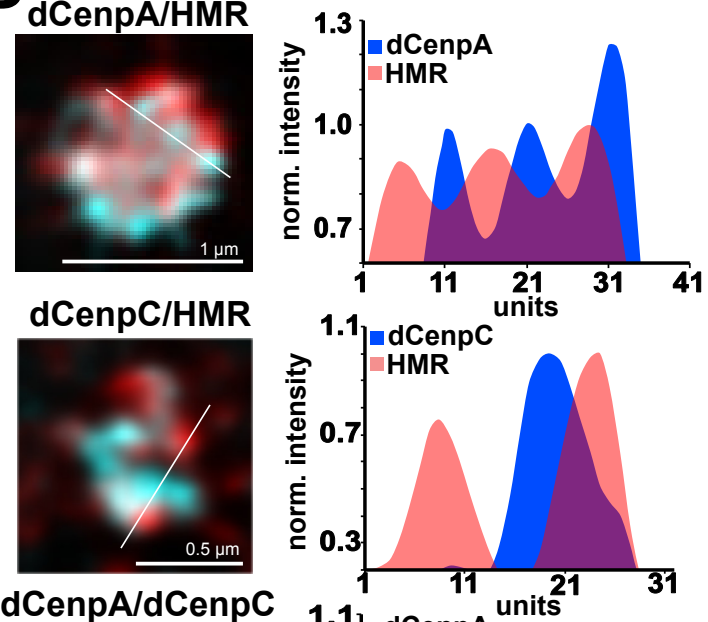

dCenpA/dCenpC

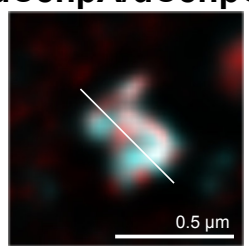


A

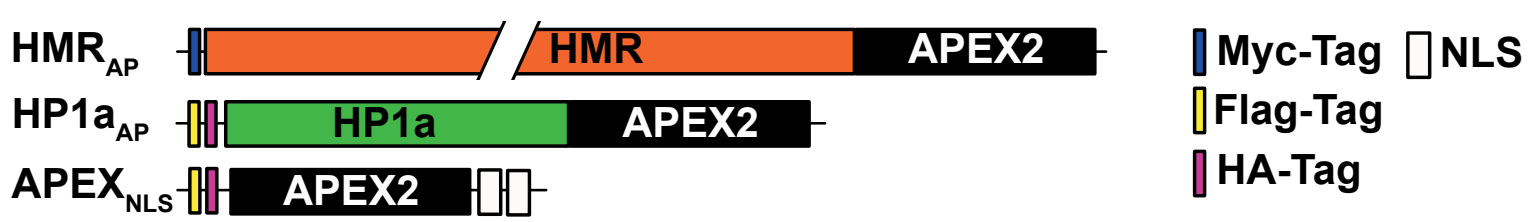
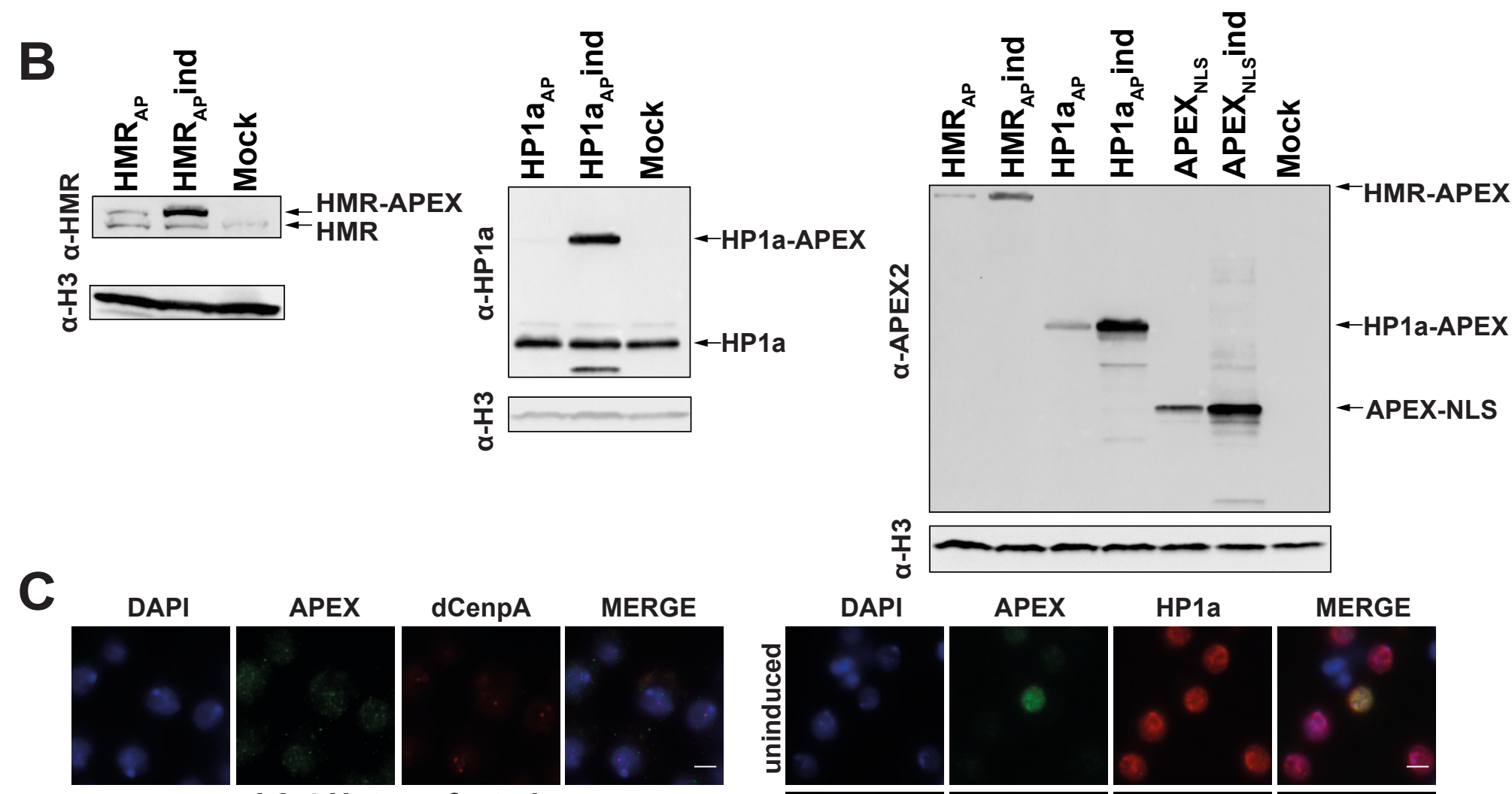

\section{L2-4 Untransfected}

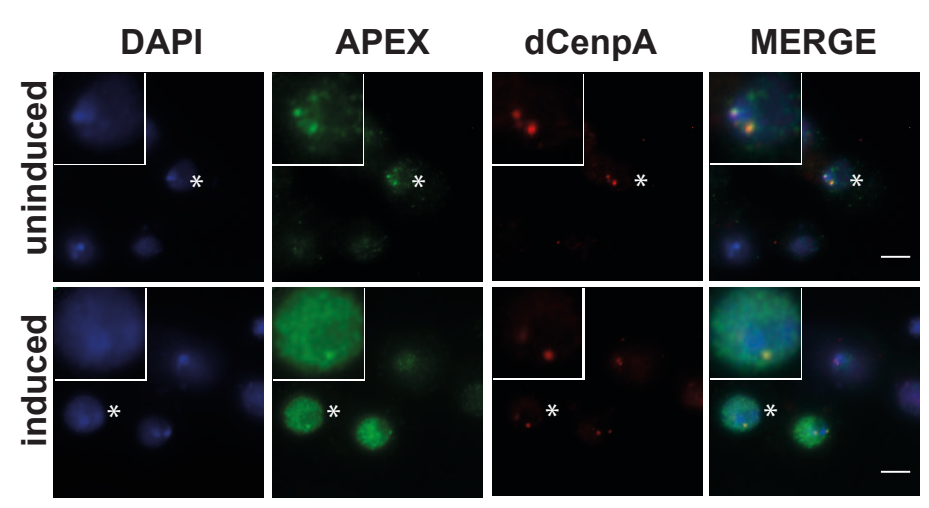

$H_{M M R_{A P}}$
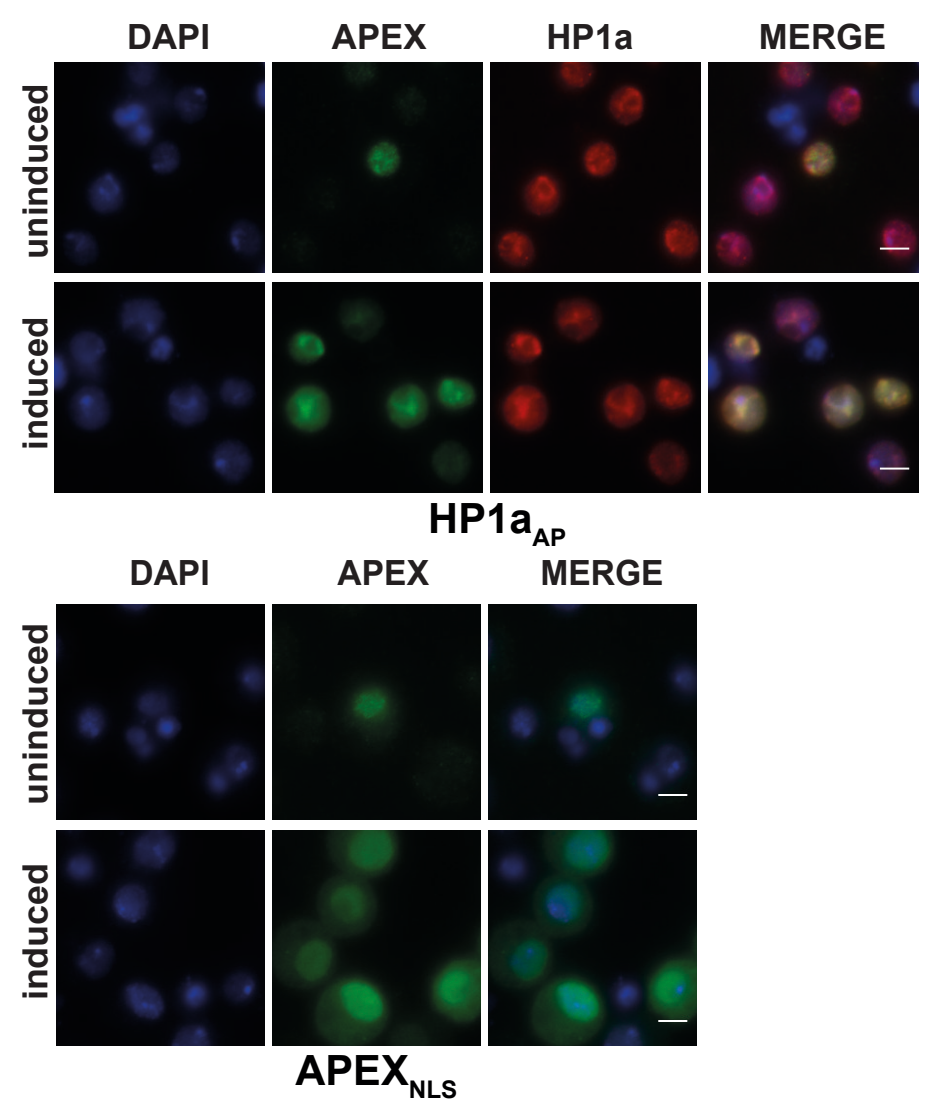

Kochanova et al., Fig. 2 
A
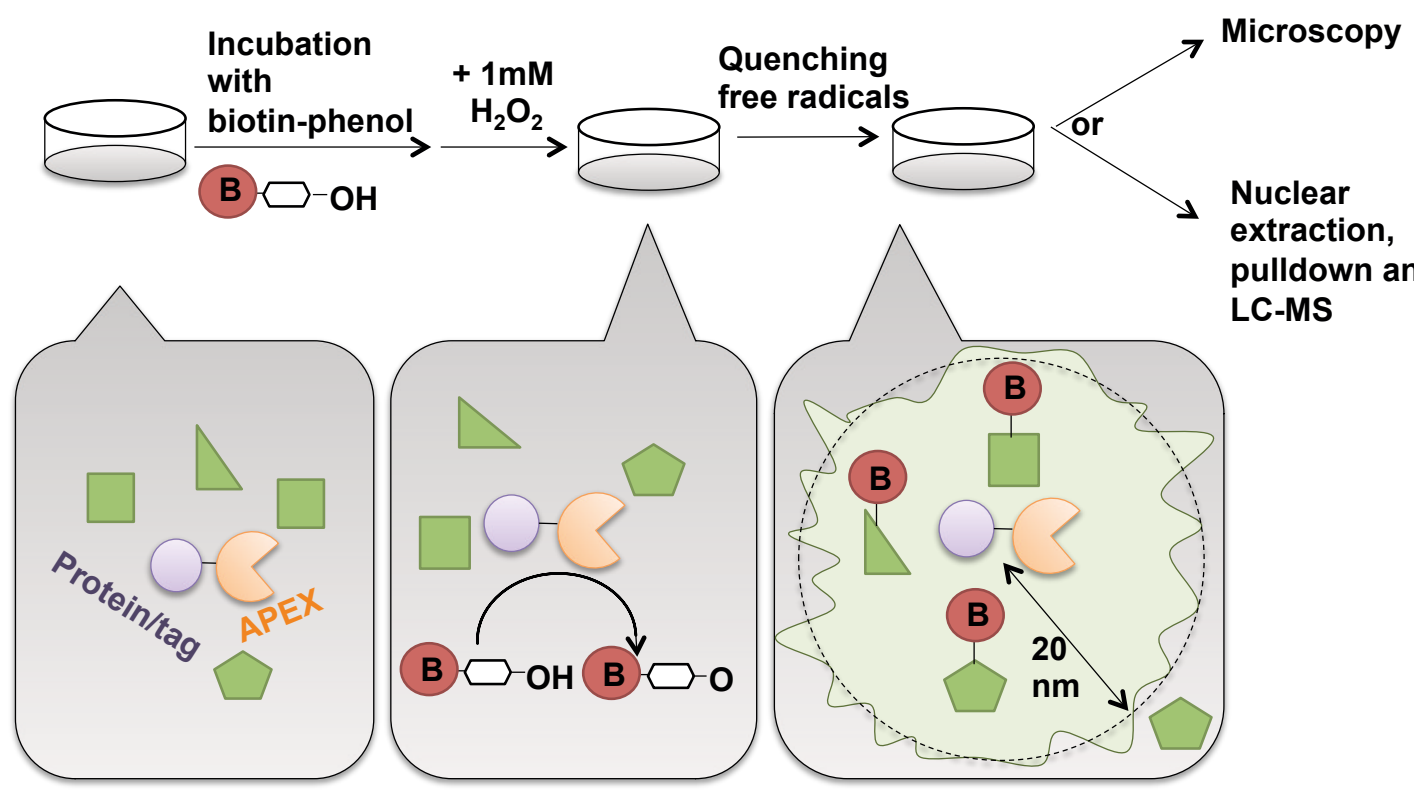

pulldown and

LC-MS
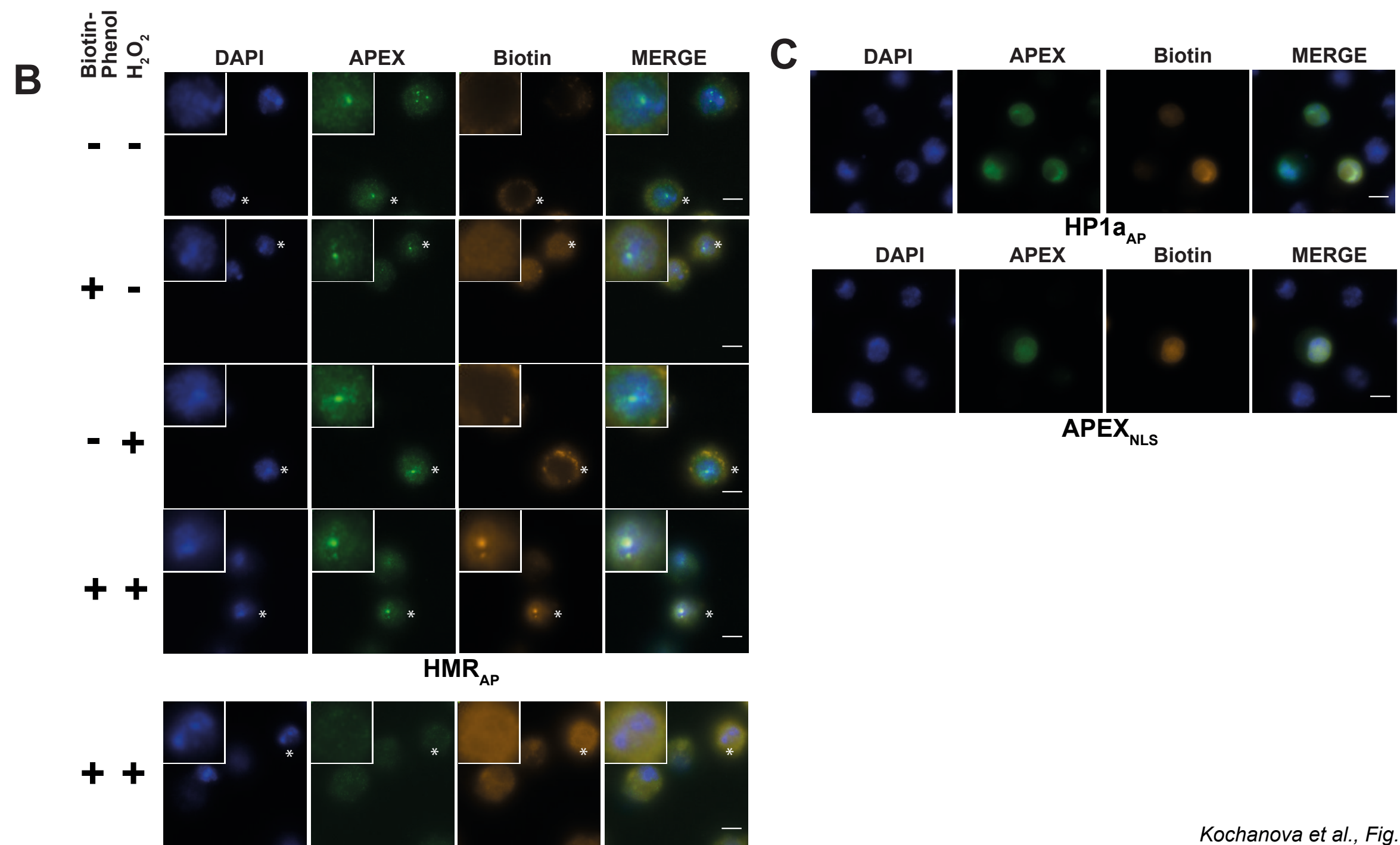

L2-4 Untransfected 

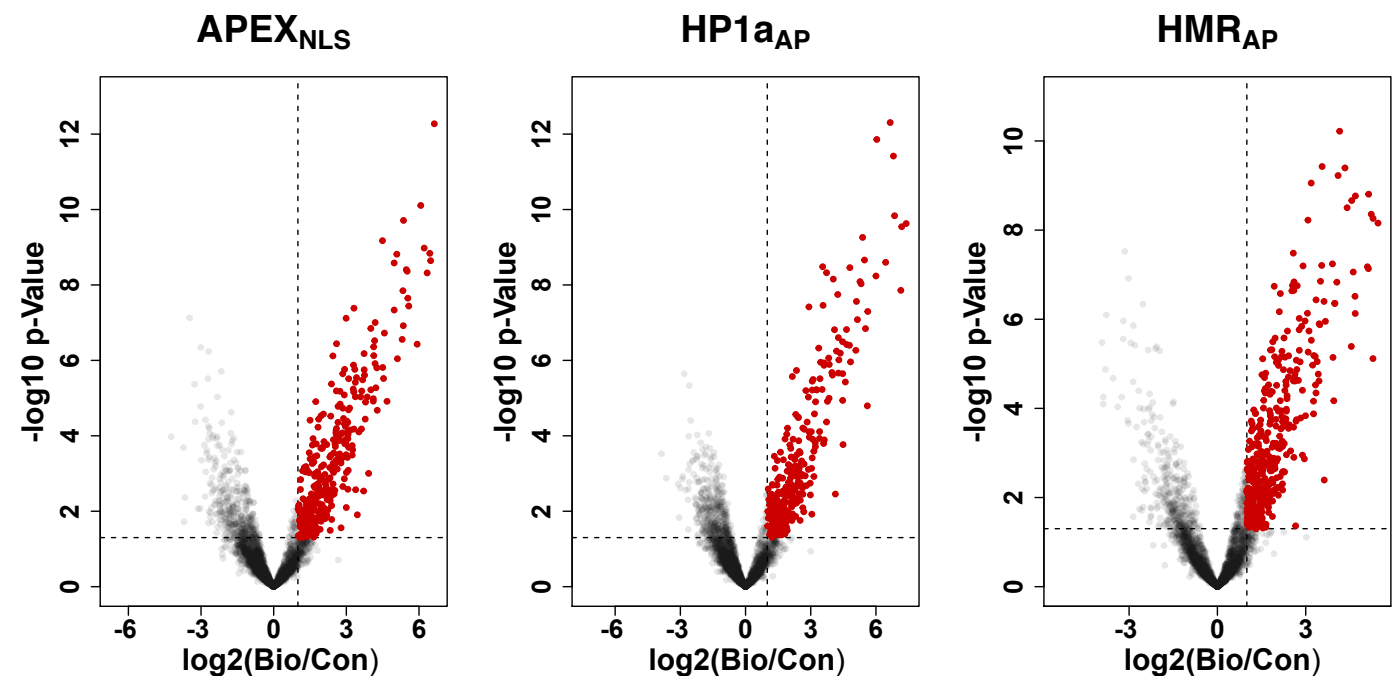

B

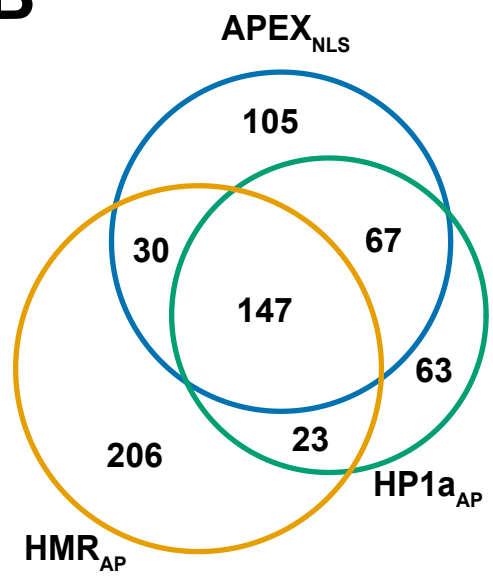

C

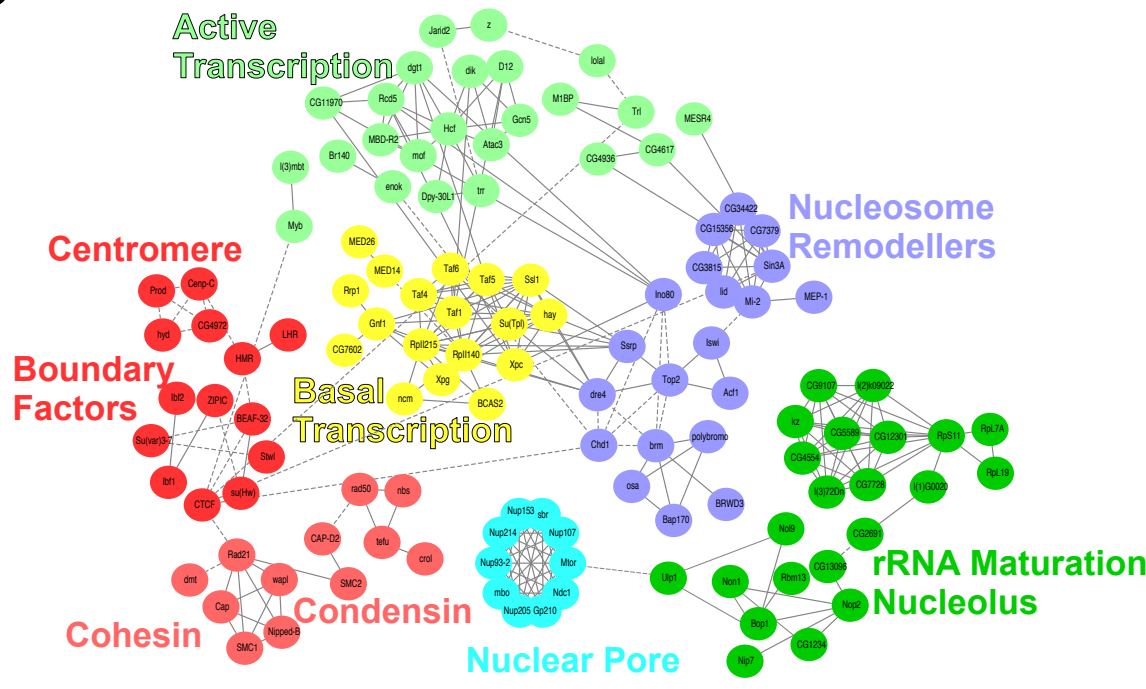


A

C

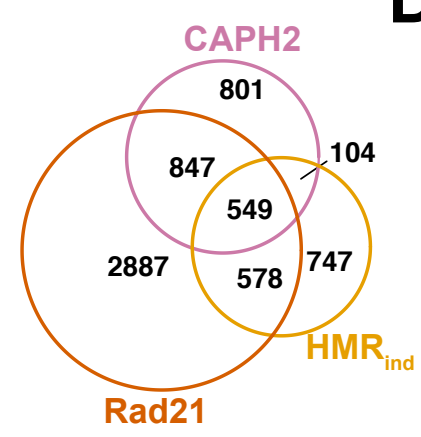

E
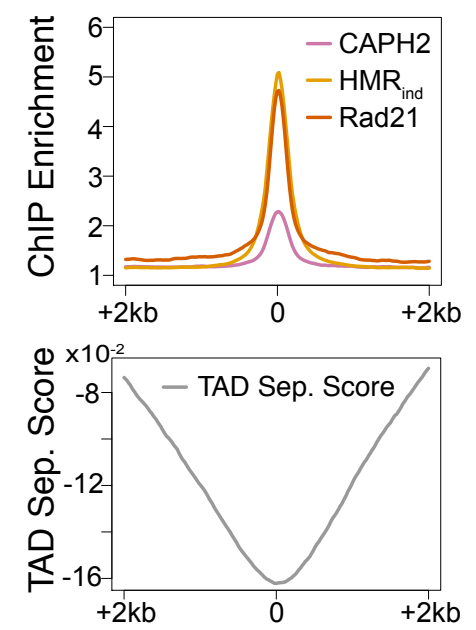

B

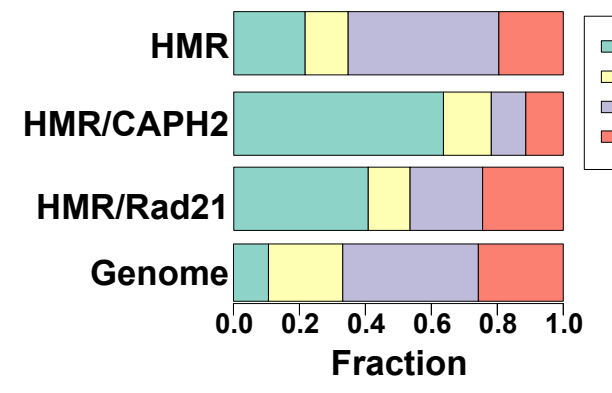

D

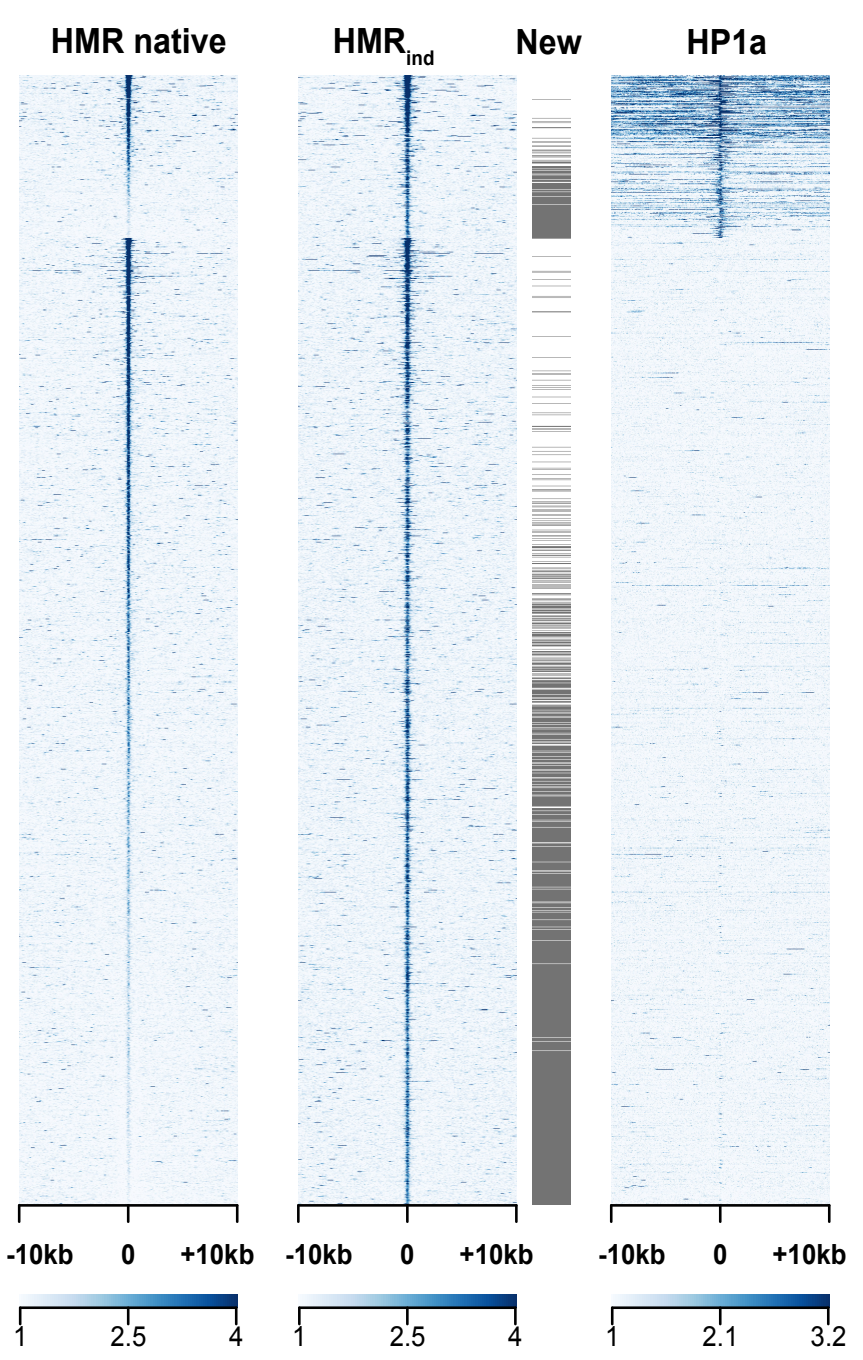

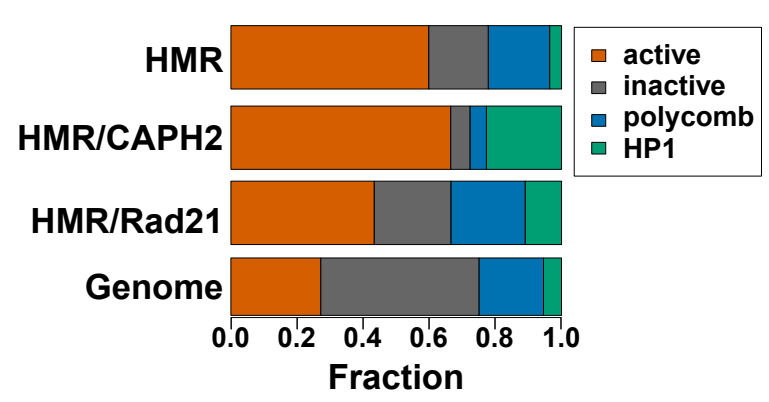

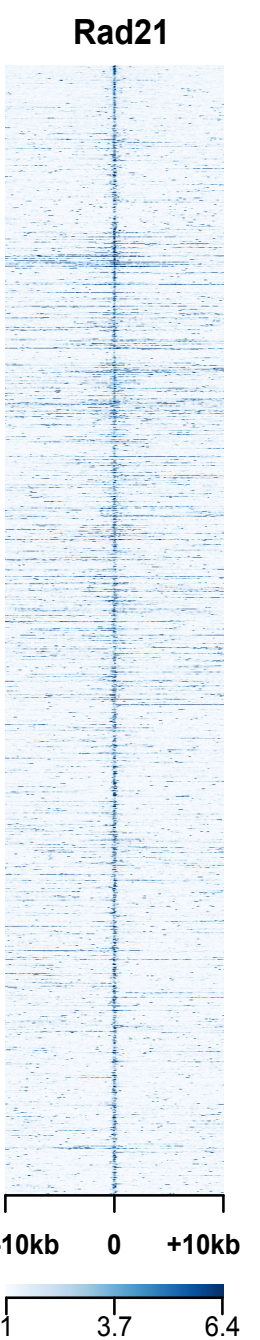

TAD Sep. Score

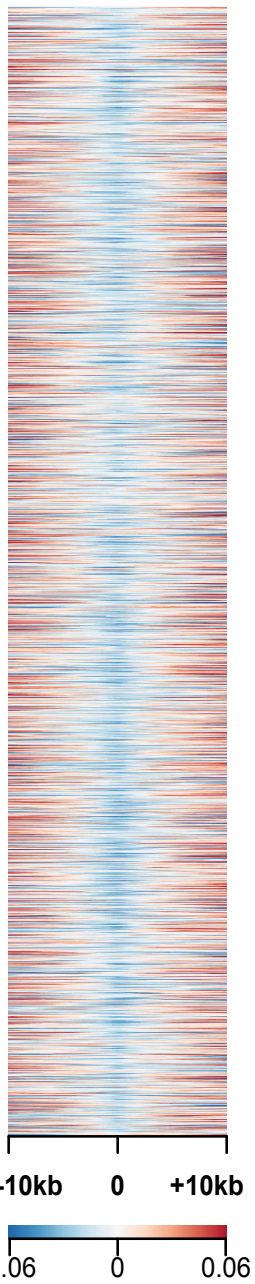




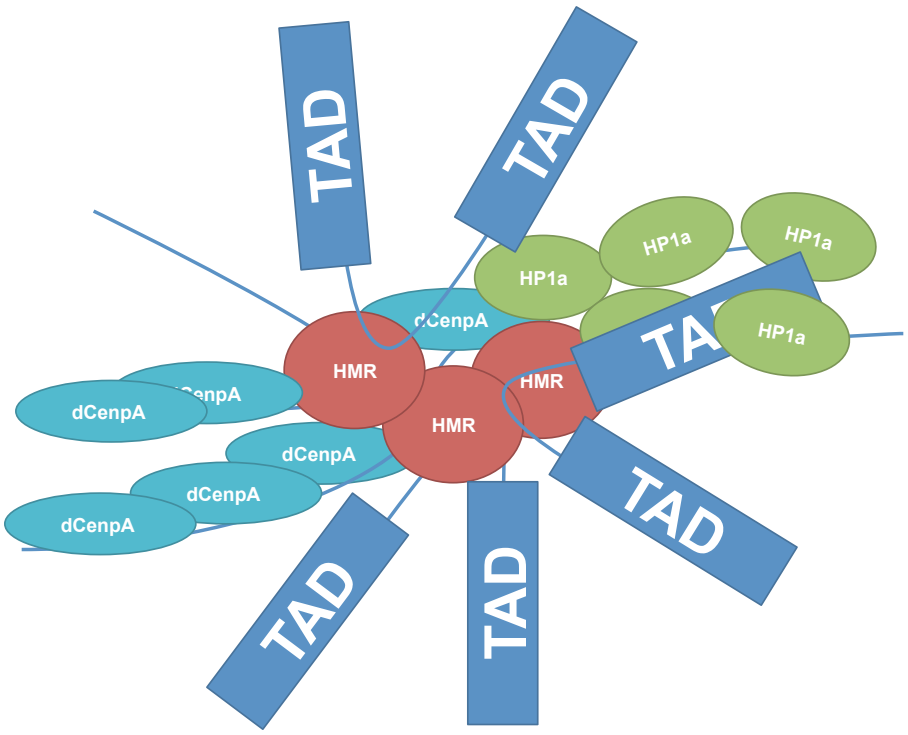

Kochanova et al., Fig. 6 